\title{
Stability and Hopf bifurcation analysis in ecological system with two delays
}

\author{
Manju Agarwal $^{1}$ \& Rachana Pathak $^{2}$ \\ Department of Mathematics \& Astronomy, Lucknow University, Lucknow-226007, INDIA \\ ${ }^{1}$ E-mail: manjuak@yahoo.com \\ ${ }^{2}$ E-mail: rachanapathak2@gmail.com
}

\begin{abstract}
This paper aims to study the effect of time-delay on a food chain model. Two delays ( $\tau_{1}$ and $\tau_{2}$ ) are considered in the model to describe the time that juveniles of prey and predator take to mature. The stability analysis of the proposed model is carried out. The Hopf bifurcation conditions of the interior equilibrium point are established. Finally, numerical simulations are done to support the analytical findings. In addition, critical value of time delays are determined and it is found that maturation delay always acts as a destabilizing factor.
\end{abstract}

Keywords: Food chain model, Time-delay, Stability, Hopf bifurcation

DOI: http://dx.doi.org/10.4314/ijest.v3i8.4

\section{Introduction}

Ecology is a highly predictive science with the goal of understanding the patterns and processes related to life on earth. The field of ecology has become the organizing principle in the effort to address the world problems because it contains both the problem and solution. The complexity of natural systems presents difficulty in finding simple answers. Science has traditionally isolated organisms in order to study them under ecology. It does not only include the community of organisms in an environment, but also the whole complex of physical factors around them. It is interested in how an organism relates within the natural community. Ecological systems are enormously complex. In an ecological system, no organism is an autonomous entity isolated from its surroundings as there are always interactions among species of the system. In ecology, biological interactions are the relationships between two species in an ecosystem. These relationships can be categorized into many different classes based either on the effects or on the mechanism of the interaction and can be classified as Neutralism, Amensalism, Competition, Parasitism and Predation. Predation describes a biological interaction where a predator (an organism that is hunting) feeds on its prey, the organism that is attacked. Predators may or may not kill their prey prior to feeding on them, but the act of predation always results in the death of the prey.

The dynamics of predator - prey model has long been and will continue to be one of the dominant themes in both ecology and mathematical ecology due to the fact that predator- prey interaction is the fundamental structure in population dynamics. In the 1920s Volterra and Lotka described a simple mathematical model of the interaction between predators and their prey by means of following nonlinear differential equations

$$
\begin{aligned}
& \dot{x}(t)=x(t)(a-b y(t)), \\
& \dot{y}(t)=-y(t)(c-d x(t)),
\end{aligned}
$$

where $y(t)$ is the density of predators (for example, foxes), $x(t)$ is the density of its prey (for example, rabbits), and $a, b, c$ and $d$ are positive constants. After the pioneering work of Lotka and Volterra, prey-predator models have been studied extensively by 
many mathematicians (Freedman, 1980; Kuang, 1990; May, 1981, 2001; Murray, 1989, 1993; Takeuchi, 1996; Turchin, 2003; Vance, 1978).

Mathematical model of multiple species interaction is the so-called food chain model. In the paper of Freedman and Waltman (1977), the authors studied the persistence of a classical (i.e. prey-dependent) three species food chain model. Chiu and Hsu (1998) discussed the extinction of top predator in a classical three-level food chain model with Michaelis-Menten functional response. Klebanoff and Hastings (1993, 1994), McCann and Yodzis (1995), Kuznetsov and Rinaldi (1996), Muratori and Rinaldi (1992) and others studied structures relevant to chaos in three species classical food chains. Freedman and So (1985) studied the global stability and persistence of a simple but general food chain model. Kuang (2001) studied similar questions for a diffusive version of that simple food chain model. Recently, Upadhyay and Vikas Rai (2001) have proposed a food chain system in the following form:

$$
\begin{aligned}
& \frac{d X}{d t}=a_{1} X-b_{1} X^{2}-\frac{w X Y}{X+D}, \\
& \frac{d Y}{d t}=-a_{2} Y+\frac{w_{1} X Y}{X+D_{1}}-\frac{w_{2} Y Z}{Y+D_{2}}, \\
& \frac{d Z}{d t}=c Z^{2}-\frac{w_{3} Z^{2}}{Y+D_{3}} .
\end{aligned}
$$

where a prey population $X$ is captured by individuals of population $Y$. This population, in turn, serves as a favorite food for individuals of population $Z . a_{1}, a_{2}, b_{1}, w, w_{1}, w_{2}, w_{3}, D, D_{1}, D_{2}, D_{3}$ and $c$ are positive constants. $a_{1}$ the intrinsic growth rate of the prey population $X, a_{2}$ the intrinsic death rate of the specialist predator $Y$ in the absence of the only food $X, C$ measures the rate of self-reproduction and the square term signifies the fact that mating frequency is directly proportional to the number of males as well as females, $D_{3}$ normalizes the residual reduction in the predator population because of severe scarcity of the favorite food, $b_{1}$ measures the effect of intra-specific competition and $D$ measures the effect of the prey in evading a predator's attack. It depends on the protection afforded by the environment to the prey. The larger the value of $D$, more elusive is the host against any attack by parasites.

However, this model is less realistic. To make model realistic one should include some of the past states of these systems; ideally real system may modeled by differential equations with time-delays. Time-delays occur so often in almost every situation, that to ignore them is to ignore reality. Time delay is the inherent property of the dynamical systems and plays an important role in almost all branches of science and particularly in biological sciences (e.g., population dynamics, epidemiology, etc.) see (Yan and Zhang, 2008; Yang, 2009; Meng et al. 2011). The importance derives from the fact that many of the phenomena around us do not act instantaneously from the moment of their occurrence. For example, a change in the resources or environment does not affect the survival of existing populations immediately. There is always a time lag between the moment an action takes place and its effect is observed. In ecology, more realistic models should include some of the past states, i.e., a real system should be modeled by differential equations with time delays. Kuang (1993) mentioned that animals take some time to digest their food before further activities and responses take place and hence any model of species dynamics without delays is an approximation at best. Now it is beyond doubt that in an improved analysis, the effect of time-delay due to the time required in going from egg stage to the adult stage, gestation period, et cetera, has to be taken into account. Detailed arguments on importance and usefulness of time-delays in realistic models may be found in the classical books of Macdonald (1989), Gopalsamy (1992) and Kuang (1993).

Keeping this in mind, we introduce maturation time delays in our model. We assume that the juveniles of prey and predator take $\tau_{1}$ and $\tau_{2}$ units of time to mature, respectively and hence our model is:

$$
\begin{aligned}
& \frac{d X}{d t}=r_{1} X\left(1-\frac{X\left(t-\tau_{1}\right)}{K_{1}}\right)-\frac{w X Y}{X+D}, \\
& \frac{d Y}{d t}=r_{2} Y\left(1-\frac{Y\left(t-\tau_{2}\right)}{K_{2}}\right)+\frac{w_{1} X Y}{X+D_{1}}-\frac{w_{2} Y Z}{Y+D_{2}}, \\
& \frac{d Z}{d t}=c Z^{2}-\frac{w_{3} Z^{2}}{Y+D_{3}} .
\end{aligned}
$$

Where $r_{i}, K_{i}, i=1,2$ are growth rate and carrying capacity of environment for prey and predator respectively.

The rest of this paper is organized as follows: In Section 2, we analyze our model with regard equilibria and their positive conditions. In Section 3, we investigate the stability of positive equilibrium and occurrence of Hopf bifurcation. In Section 4, some numerical simulations are carried out to justify the analytic results obtained in the manuscript. Section 5 deals with the conclusions of the paper. 


\section{Equilibrium analysis}

Equating the derivatives on the left hand sides to zero and solving the resulting algebraic equations, we can find following possible equilibria:

$E_{0}(0,0,0), E_{1}\left(K_{1}, 0,0\right), E_{2}\left(0, K_{2}, 0\right), E_{3}(0, \hat{y}, \hat{z}), E_{4}(\bar{x}, \bar{y}, 0), E_{5}\left(x^{*}, y^{*}, z^{*}\right)$

The equilibrium points $E_{0}(0,0,0), E_{1}\left(K_{1}, 0,0\right)$ and $E_{2}\left(0, K_{2}, 0\right)$ are always nonnegative. Now we will discuss non negativity condition for $E_{3}(0, \hat{y}, \hat{z}), E_{4}(\bar{x}, \bar{y}, 0)$ and $E_{5}\left(x^{*}, y^{*}, z^{*}\right)$.

For equilibrium points $E_{3}(0, \hat{y}, \hat{z})$ the system (1) become

$r_{2}\left(1-\frac{\hat{y}}{K_{2}}\right)-\frac{w_{2} \hat{z}}{\hat{y}+D_{2}}=0$,

$c \hat{z}^{2}-\frac{w_{3} \hat{z}^{2}}{\hat{y}+D_{3}}=0$.

Solving above algebraic system we will get

$\hat{y}=\frac{w_{3}}{c}-D_{3}, \hat{z}=\frac{r_{2}}{K_{2} w_{2}}\left(\frac{w_{3}}{c}+D_{2}-D_{3}\right)\left(K_{2}+D_{3}-\frac{w_{3}}{c}\right)$

is a non negative equilibrium point of the system (1) if

$K_{2}+D_{3}>\frac{w_{3}}{c}>D_{3}$.

For equilibrium points $E_{4}(\bar{x}, \bar{y}, 0)$ the system (1) become

$r_{1}\left(1-\frac{\bar{x}}{K_{1}}\right)-\frac{w_{2} \bar{y}}{\bar{x}+D}=0$,

$r_{2}\left(1-\frac{\bar{y}}{K_{2}}\right)+\frac{w_{1} \bar{x}}{\bar{x}+D_{1}}=0$.

Solving above algebraic system we will get

$\bar{y}=\frac{r_{1}}{w}(\bar{x}+D)\left(1-\frac{\bar{x}}{K_{1}}\right)$,

$F(\bar{x})=\frac{r_{1} r_{2}}{K_{1}} \bar{x}^{3}+\left(\frac{r_{1} r_{2} D}{K_{1}}-\frac{r_{1} r_{2} D_{1}}{K_{1}}-r_{1} r_{2}\right) \bar{x}^{2}+\left(r_{2} w K_{2}-r_{1} r_{2} D-r_{1} r_{2} D_{1}+\frac{r_{1} r_{2} D D_{1}}{K_{1}}+w w_{1} K_{2}\right) \bar{x}+$

$+D_{1} r_{2} K_{2} w-r_{1} r_{2} D D_{1}$,

$F(0)=D_{1} r_{2} K_{2} w-r_{1} r_{2} D D_{1}$,

$F(0)>0$ if $K_{2} w>r_{1} D$.

$F\left(K_{1}\right)=K_{2} w\left(r_{2} K_{1}+w_{1}+D_{1} r_{2}\right)-2 r_{1} r_{2} D_{1} K_{1}$,

$F\left(K_{1}\right)<0$ if $K_{2} w\left(r_{2} K_{1}+w_{1}+D_{1} r_{2}\right)<2 r_{1} r_{2} D_{1} K_{1}$.

We note that $F(0)>0$ and $F\left(K_{1}\right)<0$, showing the existence of $\bar{x}$ in the interval $0<\bar{x}<K_{1}$ and the sufficient condition for to be unique is $\frac{d F}{d \bar{x}}<0$ at $E_{4}$, where

$\frac{d F}{d \bar{x}}=\frac{3 r_{1} r_{2}}{K_{1}} \bar{x}^{2}+\frac{2 r_{1} r_{2}}{K_{1}}\left(D-D_{1}-K_{1}\right) \bar{x}+r_{2} w K_{2}+w w_{1} K_{2}+\frac{r_{1} r_{2} D_{1} D_{2}}{K_{1}}-r_{1} r_{2}\left(D+D_{1}\right)<0$.

Now, check the existence and uniqueness of an interior equilibrium $E_{5}\left(x^{*}, y^{*}, z^{*}\right)$ of the system (1).

Solving algebraic system (1) 


$$
\begin{aligned}
& r_{1}\left(1-\frac{x^{*}}{K_{1}}\right)-\frac{w y^{*}}{x^{*}+D}=0, \\
& r_{2}\left(1-\frac{y^{*}}{K_{2}}\right)+\frac{w_{1} x^{*}}{x^{*}+D_{1}}-\frac{w_{2} z^{*}}{y^{*}+D_{2}}=0, \\
& C Z^{*}-\frac{w_{3} z^{*}}{y^{*}+D_{3}}=0 .
\end{aligned}
$$

We will get,

$$
y^{*}=\frac{w_{3}}{c}-D_{3}, z^{*}=\frac{r_{2}}{K_{2} w_{3}}\left(\frac{w_{3}}{c}-D_{3}+D_{2}\right)\left(K_{2}-\frac{w_{3}}{c}+D_{3}\right)+\frac{w_{1} x^{*}}{x^{*}+D_{1}} \text { with } K_{2}+D_{3}>\frac{w_{3}}{c}>D_{3}
$$

and $x^{*}$ is the unique positive root of the following equation

$$
(x *)^{2}+\left(D-K_{1}\right) x^{*}+\left\{\frac{w K_{1}}{r_{1}}\left(\frac{w_{3}}{c}-D_{3}\right)-D K_{1}\right\}=0 \text {. }
$$

Conditions of the existence of the positive value $x^{*}$ are $D<K_{1}$ and $\frac{w_{3}}{c}-D_{3}<\frac{r_{1}}{w}$.

\section{Stability and local Hopf bifurcation}

We are only interested in stability of interior point. $\left(x^{*}, y^{*}, z^{*}\right)$ be the interior equilibrium of system (1). Then the linearization of Eq. (1) at $\left(x^{*}, y^{*}, z^{*}\right)$ is given by

$$
\begin{aligned}
& \frac{d x}{d t}=\left\{r_{1}\left(1-\frac{x^{*}}{K_{1}}\right)-\frac{w D y^{*}}{\left(x^{*}+D\right)^{2}}\right\} x-\frac{w y x^{*}}{x^{*}+D}-\frac{r_{1} x^{*} x\left(t-\tau_{1}\right)}{K_{1}}, \\
& \frac{d y}{d t}=\left\{r_{2}\left(1-\frac{y^{*}}{K_{2}}\right)+\frac{w_{1} x^{*}}{x^{*}+D_{1}}-\frac{D_{2} w_{2} z^{*}}{\left(y^{*}+D_{2}\right)^{2}}\right\} y+\frac{w_{1} D_{1} y^{*} x}{\left(x^{*}+D_{1}\right)^{2}}-\frac{w_{2} y^{*} z}{y^{*}+D_{2}}-\frac{r_{2} y^{*} y\left(t-\tau_{2}\right)}{K_{2}}, \\
& \frac{d z}{d t}=\frac{w_{3}\left(z^{*}\right)^{2} y}{\left(y^{*}+D_{3}\right)^{2}} .
\end{aligned}
$$

Characteristic equation is

$$
\lambda^{3}+\lambda^{2} A_{1}+\lambda E_{2}-H+e^{-\lambda \tau_{1}}\left(B_{2} \lambda^{2}+B_{1} \lambda+B_{3}\right)+e^{-\lambda \tau_{2}}\left(G_{2} \lambda^{2}+G_{1} \lambda\right)+E_{1} \lambda e^{-\lambda\left(\tau_{1}+\tau_{2}\right)}=0,
$$

where

$$
\begin{aligned}
A_{1}= & -r_{2}\left(1-\frac{y^{*}}{K_{2}}\right)-\frac{w_{1} x^{*}}{x^{*}+D_{1}}+\frac{D_{2} w_{2} z^{*}}{\left(y^{*}+D_{2}\right)^{2}}-r_{1}\left(1-\frac{x^{*}}{K_{1}}\right)+\frac{w D y^{*}}{\left(x^{*}+D\right)^{2}}, \\
B_{1}= & -\frac{r_{1} r_{2} x^{*}}{K_{1}}\left(1-\frac{y^{*}}{K_{2}}\right)-\frac{r_{1}\left(x^{*}\right)^{2} w_{1}}{K_{1}\left(x^{*}+D_{1}\right)}+\frac{r_{1} x^{*} D_{2} w_{2} z^{*}}{K_{1}\left(y^{*}+D_{2}\right)^{2}}, \\
H= & \frac{w_{2} w_{3} y^{*}\left(z^{*}\right)^{2}}{\left(y^{*}+D_{2}\right)\left(y^{*}+D_{3}\right)^{2}}\left\{r_{1}\left(1-\frac{x^{*}}{K_{1}}\right)-\frac{D w y^{*}}{\left(x^{*}+D\right)^{2}}\right\}, B_{3}=\frac{r_{1} w_{2} w_{3} x^{*} y^{*}\left(z^{*}\right)^{2}}{K_{1}\left(y^{*}+D_{2}\right)\left(y^{*}+D_{3}\right)^{2}}, \\
B_{2}= & \frac{r_{1} x^{*}}{K_{1}}, G_{2}=\frac{r_{2} y^{*}}{K_{2}}, E_{1}=\frac{r_{1} r_{2} x^{*} y^{*}}{K_{1} K_{2}}, G_{1}=-\frac{r_{1} r_{2} y^{*}}{K_{2}}\left(1-\frac{x^{*}}{K_{1}}\right)+\frac{w D r_{2}\left(y^{*}\right)^{2}}{K_{2}\left(x^{*}+D\right)^{2}}, \\
E_{2}= & r_{1}\left(1-\frac{x^{*}}{K_{1}}\right)\left\{r_{2}\left(1-\frac{y^{*}}{K_{2}}\right)+\frac{w_{1} x^{*}}{x^{*}+D_{1}}-\frac{D_{2} w_{2} z^{*}}{\left(y^{*}+D_{2}\right)^{2}}\right\}-r_{2}\left(1-\frac{y^{*}}{K_{2}}\right)\left(\frac{w D y^{*}}{\left(x^{*}+D\right)^{2}}\right)-\frac{w w_{1} D x^{*} y^{*}}{\left(x^{*}+D\right)^{2}\left(x^{*}+D_{1}\right)} \\
& +\frac{w w_{2} D D_{2} y^{*} z^{*}}{\left(x^{*}+D\right)^{2}\left(y^{*}+D_{2}\right)^{2}}+\frac{w w_{1} D_{1} x^{*} y^{*}}{\left(x^{*}+D\right)\left(x^{*}+D_{1}\right)^{2}}+\frac{w_{2} w_{3} y^{*}\left(z^{*}\right)^{2}}{\left(y^{*}+D_{2}\right)\left(y^{*}+D_{3}\right)^{2}} .
\end{aligned}
$$




\section{Stability of the system:}

Case A: $\tau_{1} \neq \tau_{2}$

Sub case 1: $\tau_{1}>0, \tau_{2}>0$

We consider Eq. (3.2) with $\tau_{2}$ in its stable interval and $\tau_{1}$ as a parameter. Let $\lambda=i \delta$ be one such root. Substituting this in equation (3.2) and equating real and imaginary parts, we get

$$
\begin{gathered}
\cos \delta \tau_{1}\left\{B_{1} \delta+E_{1} \delta \cos \delta \tau_{2}\right\}-\sin \delta \tau_{1}\left\{-B_{2} \delta^{2}+B_{3}+\delta E_{1} \sin \delta \tau_{2}\right\}=\delta^{3}-\delta E_{2}-G_{1} \delta \cos \delta \tau_{2} \\
-G_{2} \delta^{2} \sin \delta \tau_{2}, \\
\cos \delta \tau_{1}\left\{-B_{2} \delta^{2}+B_{3}+\delta E_{1} \sin \delta \tau_{2}\right\}+\sin \delta \tau_{1}\left\{B_{1} \delta+E_{1} \delta \cos \delta \tau_{2}\right\}=\delta^{2} A_{1}+H+G_{2} \delta^{2} \cos \delta \tau_{2} \\
-G_{1} \delta \sin \delta \tau_{2} .
\end{gathered}
$$

Squaring and adding equations (3.3a) and (3.3b), we get

$\delta^{6}+\bar{A} \delta^{4}+\bar{B} \delta^{2}+\bar{S} \sin \delta \tau_{2}+H^{2}-B_{3}^{2}=0$,

where

$$
\begin{aligned}
& \bar{A}=-B_{2}^{2}-2 E_{2}+G_{2}^{2}-2 G_{1} \cos \delta \tau_{2}+A_{1}^{2}+2 G_{2} A_{1} \cos \delta \tau_{2}, \\
& \bar{B}=E_{2}^{2}+G_{1}^{2}+2 E_{2} G_{1} \cos \delta \tau_{2}+2 A_{1} H+2 H G_{2} \cos \delta \tau_{2}-B_{1}^{2}-E_{1}^{2}-2 B_{1} E_{1} \cos \delta \tau_{2}+2 B_{2} B_{3}, \\
& \bar{S}=-2 G_{1} \delta^{5}+2 E_{2} G_{2} \delta^{3}-2 A_{1} G_{1} \delta^{3}-2 H G_{1} \delta-2 B_{3} E_{1} \delta+2 B_{2} E_{1} \delta^{3} .
\end{aligned}
$$

We define $F(\delta)=\delta^{6}+\bar{A} \delta^{4}+\bar{B} \delta^{2}+\bar{S} \sin \delta \tau_{2}+H^{2}-B_{3}^{2}$ and assume that:

$\left(\mathrm{H}_{1}\right): F(0)=H<B_{3}$

holds. It is easy to check that $F(0)<0$ if $\left(\mathrm{H}_{1}\right)$ holds and $F(\infty)>0$. Therefore, the equation (3.4) always has at least one positive root.

Sub case 2: $\tau_{1}=0, \tau_{2}>0$

The associated characteristic equation of system (3.2) is

$$
\lambda^{3}+\lambda^{2}\left(A_{1}+B_{2}\right)+\lambda\left(B_{1}+E_{2}\right)+B_{3}-H+e^{-\lambda \tau_{2}}\left\{G_{2} \lambda^{2}+\lambda\left(G_{1}+E_{1}\right)\right\}=0 \text {. }
$$

Let $\lambda=i \delta$ be one such root. Substituting this in equation (3.5) and equating real and imaginary parts, we get

$\delta\left(G_{1}+E_{1}\right) \cos \delta \tau_{2}+G_{2} \delta^{2} \sin \delta \tau_{2}=\delta^{3}-\delta\left(B_{1}+E_{2}\right)$,

$\delta\left(G_{1}+E_{1}\right) \sin \delta \tau_{2}-G_{2} \delta^{2} \cos \delta \tau_{2}=\delta^{2}\left(A_{1}+B_{2}\right)+H-B_{3}$.

Squaring and adding equations (3.5a) and (3.5b), we get

$\delta^{6}+A^{*} \delta^{4}+B^{*} \delta^{2}+\left(H-B_{3}\right)^{2}=0$,

where

$$
\begin{aligned}
& A^{*}=\left(A_{1}+B_{2}\right)^{2}-G_{2}^{2}-2\left(B_{1}+E_{2}\right), \\
& B^{*}=\left(B_{1}+E_{2}\right)^{2}+2\left(H-B_{3}\right)\left(A_{1}+B_{2}\right)-\left(G_{1}+E_{1}\right)^{2} .
\end{aligned}
$$

Substituting $\delta^{2}=\sigma$ equation (3.6) becomes

$F(\sigma)=\sigma^{3}+A^{*} \sigma^{2}+B^{*} \sigma+\left(H-B_{3}\right)^{2}=0$,

$F(0)=\left(H-B_{3}\right)^{2}$.

We notice that $F$ is continuous everywhere with $F(0)>0$ when condition $\left(\mathrm{H}_{1}\right)$ holds and $F(\infty)<0$. Therefore, the equation (3.7) always has at least one positive root. Consequently, the stability criteria of the system for $\tau=0$ will not necessarily ensure the stability of the system for $\tau \neq 0$.

We assume the equation (3.7) has three positive roots denoted by $\sigma_{1}, \sigma_{2}, \sigma_{3}$ respectively which are as:

$\delta_{1}=\sqrt{\sigma_{1}}, \delta_{2}=\sqrt{\sigma_{2}}, \delta_{3}=\sqrt{\sigma_{3}}$.

Again solving (3.5a) and (3.5b), we get a critical value of delay given as follows 
$\tau_{2 K}=\frac{1}{\delta} \cos ^{-1}\left[\frac{\delta^{2}\left\{G_{1}+E_{1}-G_{2}\left(A_{1}+B_{2}\right)\right\}-\left(B_{1}+E_{2}\right)\left(G_{1}+E_{1}\right)-G_{2}\left(H-B_{3}\right)}{\left(G_{1}+E_{1}\right)^{2}+G_{2}^{2} \delta^{2}}\right]+\frac{2 k \pi}{\delta}, \quad k=0,1,2, \ldots \ldots$

Sub case 3: $\tau_{1}>0, \tau_{2}=0$

The associated characteristic equation of system (3.2) is

$\lambda^{3}+\lambda^{2}\left(A_{1}+G_{2}\right)+\lambda\left(E_{2}+G_{1}\right)-H+e^{-\lambda \tau_{1}}\left\{B_{2} \lambda^{2}+\lambda\left(B_{1}+E_{1}\right)+B_{3}\right\}=0$.

Let $\lambda=i \delta$ be one such root. Substituting this in equation (3.8) and equating real and imaginary parts, we get

$\delta\left(B_{1}+E_{1}\right) \cos \delta \tau_{1}-\left(-B_{2} \delta^{2}+B_{3}\right) \sin \delta \tau_{1}=\delta^{3}-\delta\left(E_{2}+G_{1}\right)$,

$\delta\left(B_{1}+E_{1}\right) \sin \delta \tau_{1}+\cos \delta \tau_{1}\left(-B_{2} \delta^{2}+B_{3}\right)=\delta^{2}\left(A_{1}+G_{2}\right)+H$.

Squaring and adding equations (3.8a) and (3.8b), we get

$\delta^{6}+A^{* *} \delta^{4}+B^{* *} \delta^{2}+H^{2}-B_{3}^{2}=0$,

where

$$
\begin{aligned}
& A^{* *}=\left(A_{1}+G_{2}\right)^{2}-B_{2}^{2}-2\left(G_{1}+E_{2}\right), \\
& B^{* *}=E_{2}+G_{1}+2 H\left(A_{1}+G_{2}\right)-\left(B_{1}+E_{1}\right)^{2}+2 B_{2} B_{3} .
\end{aligned}
$$

Substituting $\delta^{2}=\sigma$ equation (3.9) becomes

$$
\begin{aligned}
& F(\sigma)=\sigma^{3}+A^{* *} \sigma^{2}+B^{* *} \sigma+H^{2}-B_{3}^{2}=0, \\
& F(0)=H^{2}-B_{3}^{2} .
\end{aligned}
$$

We notice that $F$ is continuous everywhere with $F(0)<0$ when condition $\left(\mathrm{H}_{1}\right)$ holds and $F(\infty)>0$. Therefore, the equation (3.10) always has at least one positive root. Consequently, the stability criteria of the system for $\tau=0$ will not necessarily ensure the stability of the system for $\tau \neq 0$. We assume the equation (3.10) has three positive roots denoted by $\sigma_{1}, \sigma_{2}, \sigma_{3}$ denoted as:

$\delta_{1}=\sqrt{\sigma_{1}}, \delta_{2}=\sqrt{\sigma_{2}}, \delta_{3}=\sqrt{\sigma_{3}}$.

Again solving (3.8a) and (3.8b), we get a critical value of delay given as follows

$\tau_{1 \kappa}=\frac{1}{\delta} \cos ^{-1}\left[\frac{\delta^{4}\left\{B_{1}+E_{1}-B_{2}\left(A_{1}+G_{2}\right)\right\}+\delta^{2}\left\{B_{3}\left(A_{1}+G_{2}\right)-\left(E_{2}+G_{1}\right)\left(B_{1}+E_{1}\right)-B_{2} H\right\}+B_{3} H}{B_{2}^{2} \delta^{4}+\delta^{2}\left\{\left(B_{1}+E_{1}\right)^{2}-2 B_{2} B_{3}\right\}+B_{3}^{2}}\right]+\frac{2 k \pi}{\delta}, \quad k=0,1,2, \ldots . .$.

Case B: $\tau_{1}=\tau_{2}$

Sub case 4: $\tau_{1}=\tau_{2}=\tau>0$

The associated characteristic equation of system (3.2) is

$\lambda^{3}+\lambda^{2} A_{1}+\lambda E_{2}-H+e^{-\lambda \tau}\left\{\left(B_{2}+G_{2}\right) \lambda^{2}+\lambda\left(B_{1}+G_{1}\right)+B_{3}\right\}+E_{1} \lambda e^{-2 \lambda \tau}=0$.

Multiplying $e^{\lambda \tau}$ on both sides of (3.11), it is obvious to obtain

$e^{\lambda \tau}\left(\lambda^{3}+\lambda^{2} A_{1}+\lambda E_{2}-H\right)+\left(B_{2}+G_{2}\right) \lambda^{2}+\lambda\left(B_{1}+G_{1}\right)+B_{3}+E_{1} \lambda e^{-\lambda \tau}=0$.

Let $\lambda=i \delta$ be one such root. Substituting this in equation (3.12) and equating real and imaginary parts, we get

$\cos \delta \tau\left(\delta E_{2}-\delta^{3}+E_{1} \delta\right)-\sin \delta \tau\left(\delta^{2} A_{1}+H\right)=-\left(\mathrm{B}_{1}+G_{1}\right) \delta$,

$\cos \delta \tau\left(\delta^{2} A_{1}+H\right)+\sin \delta \tau\left(\delta E_{2}-\delta^{3}-E_{1} \delta\right)=B_{3}-B_{2} \delta^{2}-G_{2} \delta^{2}$.

It follows from (3.12a) and (3.12b) that

$\sin \delta \tau=\frac{\left\{B_{3}-\left(B_{2}+G_{2}\right) \delta^{2}\right\}\left(\delta E_{2}-\delta^{3}+E_{1} \delta\right)+\left(B_{1}+G_{1}\right)\left(\delta^{3} A_{1}+H \delta\right)}{\left(\delta E_{2}-\delta^{3}\right)^{2}-E_{1}^{2} \delta^{2}+\left(\delta^{2} A_{1}+H\right)^{2}}$,
$\cos \delta \tau=\frac{\delta^{2}\left(E_{1}-E_{2}\right)\left(B_{1}+G_{1}\right)+\delta^{4}\left\{\begin{array}{l}B_{1}+G_{1} \\ -A_{1}\left(B_{2}+G_{2}\right)\end{array}\right\}+\delta^{2} B_{3} A_{1}-H \delta^{2}\left(B_{2}+G_{2}\right)+B_{3} H}{\left(\delta E_{2}-\delta^{3}\right)^{2}-E_{1}^{2} \delta^{2}+\left(\delta^{2} A_{1}+H\right)^{2}}$.

As is known to all, $\sin ^{2} \delta \tau+\cos ^{2} \delta \tau=1$. So we have

$\delta^{12}+T_{1} \delta^{10}+T_{2} \delta^{8}+T_{3} \delta^{6}+T_{4} \delta^{4}+T_{5} \delta^{2}+T_{6}=0$,

where 


$$
\begin{aligned}
T_{1}= & 2 A_{1}^{2}-B_{2}^{2}-4 E_{2}-2 B_{2} G_{2}-G_{2}^{2}, \\
T_{2}= & 4 A_{1} H+2 E_{2} G_{2}^{2}+2 E_{1} G_{2}^{2}-A_{1}^{2} G_{2}^{2}+4 B_{2} E_{2} G_{2}+4 B_{2} E_{1} G_{2}+2 B_{3} G_{2}-2 A_{1}^{2} B_{2} G_{2}-G_{1}^{2}-2 B_{1} G_{1}+ \\
& 6 E_{2}^{2}+2 B_{2}^{2} E_{2}-4 A_{1}^{2} E_{2}-2 E_{1}^{2}+A_{1}^{4}-B_{1}^{2}-A_{1}^{2} B_{2}^{2}+2 B_{2} B_{3}+2 B_{2}^{2} E_{1}, \\
T_{3}= & 2 A_{1}^{2} B_{2} B_{3}-A_{1}^{2} B_{1}^{2}-B_{3}^{2}-2 B_{1}^{2} E_{1}+4 A_{1} B_{1} B_{2} E_{1}-4 B_{2} B_{3} E_{1}-2 A_{1}^{2} E_{1}^{2}-B_{2}^{2} E_{1}^{2}+2 B_{1}^{2} E_{2}-4 B_{2} B_{3} E_{2}- \\
& 2 B_{2}^{2} E_{1} E_{2}+4 E_{1}^{2} E_{2}+2 A_{1}^{2} E_{2}^{2}-B_{2}^{2} E_{2}^{2}-4 E_{2}^{3}-2 A_{1}^{2} B_{1} G_{1}-4 B_{1} E_{1} G_{1}+4 A_{1} B_{2} E_{1} G_{1}+ \\
& 4 B_{1} E_{2} G_{1}-A_{1}^{2} G_{1}^{2}-2 E_{1} G_{1}^{2}+2 E_{2} G_{1}^{2}+2 A_{1}^{2} B_{3} G_{2}+4 A_{1} B_{1} E_{1} G_{2}-4 B_{3} E_{1} G_{2}-2 B_{2} E_{1}^{2} G_{2}- \\
& 4 B_{3} E_{2} G_{2}-4 B_{2} E_{1} E_{2} G_{2}-2 B_{2} E_{2}^{2} G_{2}+4 A_{1} E_{1} G_{1} G_{2}-E_{1}^{2} G_{2}^{2}-2 E_{1} E_{2} G_{2}^{2}-E_{2}^{2} G_{2}^{2}+4 A_{1}^{3} H-2 A_{1} B_{2}^{2} H- \\
& 8 A_{1} E_{2} H-4 A_{1} B_{2} G_{2} H-2 A_{1} G_{2}^{2} H+2 H^{2},
\end{aligned}
$$

$$
\begin{aligned}
T_{4}= & 2 B_{3}^{2} E_{1}-A_{1}^{2} B_{3}^{2}-4 A_{1} B_{1} B_{3} E_{1}+B_{1}^{2} E_{1}^{2}+2 B_{2} B_{3} E_{1}^{2}+E_{1}^{4}+2 B_{3}^{2} E_{2}+2 B_{1}^{2} E_{1} E_{2}+4 B_{2} B_{3} E_{1} E_{2}-B_{1}^{2} E_{2}^{2}+2 B_{2} B_{3} E_{2}^{2} \\
& -2 E_{1}^{2} E_{2}^{2}+E_{2}^{4}-4 A_{1} B_{3} E_{1} G_{1}-2 B_{1} E_{1}^{2} G_{1}+4 B_{1} E_{1} E_{2} G_{1}-2 B_{1} E_{2}^{2} G_{1}-E_{1}^{2} G_{1}^{2}+2 E_{1} E_{2} G_{1}^{2}-E_{2}^{2} G_{1}^{2}+2 B_{3} E_{1}^{2} G_{2} \\
& +4 B_{3} E_{1} E_{2} G_{2}+2 B_{3} E_{2}^{2} G_{2}-2 A_{1} B_{1}^{2} H+4 A_{1} B_{2} B_{3} H+4 B_{1} B_{2} E_{1} H-4 A_{1} E_{1}^{2} H+4 A_{1} E_{2}^{2} H-4 A_{1} B_{1} G_{1} H+ \\
& 4 B_{2} E_{1} G_{1} H-2 A_{1} G_{1}^{2} H+4 A_{1} B_{3} G_{2} H+4 B_{1} E_{1} G_{2} H+4 E_{1} G_{1} G_{2} H+6 A_{1}^{2} H^{2}-B_{2}^{2} H^{2}-4 E_{2} H^{2}-2 B_{2} G_{2} H^{2} \\
& -G_{2}^{2} H^{2}
\end{aligned}
$$$$
T_{5}=2 B_{3} G_{2} H^{2}+4 A_{1} H^{3}-G_{1}^{2} H^{2}-2 B_{1} G_{1} H^{2}+2 E_{2}^{2} H^{2}-2 E_{1}^{2} H^{2}+2 B_{2} B_{3} H^{2}-B_{1}^{2} H^{2}-4 B_{3} E_{1} G_{1} H-4 B_{1} B_{3} E_{1} H-
$$$$
2 A_{1} B_{3}^{2} H-B_{3}^{2} E_{2}^{2}-2 B_{3}^{2} E_{1} E_{2}-B_{3}^{2} E_{1}^{2},
$$$$
T_{6}=H^{4}-B_{3}^{2} H^{2} \text {. }
$$

Substituting $\delta^{2}=\sigma$ equation (3.15) becomes

$$
\begin{aligned}
& F(\sigma)=\sigma^{6}+T_{1} \sigma^{5}+T_{2} \sigma^{4}+T_{3} \sigma^{3}+T_{4} \sigma^{2}+T_{5} \sigma+T_{6}=0, \\
& F(0)=H^{4}-B_{3}^{2} H^{2} .
\end{aligned}
$$

We notice that $F$ is continuous everywhere with $F(0)<0$ when condition $\left(\mathrm{H}_{1}\right)$ holds and $F(\infty)>0$. Therefore, the equation (3.15) always has at least one positive root. Consequently, the stability criteria of the system for $\tau=0$ will not necessarily ensure the stability of the system for $\tau \neq 0$. We assume the equation (3.15) has six positive roots $\delta_{i}=\sqrt{\sigma_{i}}, i=1,2, \ldots . .6$.

From (3.13b), we get a critical value of delay that is given as follows

$$
\begin{aligned}
\tau_{k}= & \frac{1}{\delta} \cos ^{-1}\left[\frac{\delta^{2}\left(E_{1}-E_{2}\right)\left(B_{1}+G_{1}\right)+\delta^{4}\left\{\begin{array}{l}
B_{1}+G_{1} \\
\left.-A_{1}\left(B_{2}+G_{2}\right)\right\}
\end{array}\right\}+\delta^{2} B_{3} A_{1}-H \delta^{2}\left(B_{2}+G_{2}\right)+B_{3} H}{\left(\delta E_{2}-\delta^{3}\right)^{2}-E_{1}^{2} \delta^{2}+\left(\delta^{2} A_{1}+H\right)^{2}}\right] \\
& +\frac{2 k \pi}{\delta}, \quad k=0,1,2, \ldots . . .
\end{aligned}
$$

Sub case 5: $\tau_{1}=\tau_{2}=0$.

The associated characteristic equation of system (3.2) is

$$
\lambda^{3}+M_{1} \lambda^{2}+M_{2} \lambda+M_{3}=0 \text {, }
$$

where

$$
\begin{aligned}
& M_{1}=A_{1}+B_{2}+G_{2}, \\
& M_{2}=B_{1}+E_{2}+G_{1}+E_{1}, \\
& M_{3}=B_{3}-H .
\end{aligned}
$$

By Routh-Hurwitz's condition, all roots of Eq. (3.17) have negative real parts if and only if $\left(\mathrm{H}_{5}\right): M_{1}>0, \mathrm{M}_{3}>0$ and $\Delta=M_{1} M_{2}-M_{3}>0$ 
So the equilibrium point $\left(x^{*}, y^{*}, z^{*}\right)$ is locally asymptotically stable when $\left(\mathrm{H}_{1}\right)$ holds and unstable if either of these conditions is not satisfied.

\section{Hopf bifurcation}

We observe that the conditions for Hopf bifurcation (Hale and Lunel, (1993)) are satisfied yielding the required periodic solution, that is,

$$
\left[\frac{d(\operatorname{Re} \lambda)}{d \tau}\right]_{\tau=\tau_{0}} \neq 0
$$

This signifies that there exists at least one eigenvalue with positive real part for $\tau>\tau_{0}$. We have studied Hopf bifurcation for two cases, namely (i) $\tau_{1}=0, \tau_{2}>0$, (ii) $\tau_{1}=\tau_{2}=\tau>0$. We are not interested in case 5 , case 3 is like case 2 and it is difficult to find hopf bifurcation condition of case 1 so it is ignored.

Case 1: $\tau_{1}=0, \tau_{2}>0$

Differentiating equation (3.5) with respect to $\tau_{2}$, we obtain

$$
\begin{aligned}
{\left[3 \lambda^{2}+2 \lambda\left(A_{1}+B_{2}\right)+B_{1}+E_{2}-\tau_{2} e^{-\lambda \tau_{2}}\left\{G_{2} \lambda^{2}+\lambda\left(E_{1}+G_{1}\right)\right\}\right.} & \left.+e^{-\lambda \tau_{2}}\left(2 \lambda G_{2}+E_{1}+G_{1}\right)\right] \frac{d \lambda}{d \tau_{2}} \\
& =\lambda e^{-\lambda \tau_{2}}\left\{G_{2} \lambda^{2}+\lambda\left(E_{1}+G_{1}\right)\right\},
\end{aligned}
$$

therefore,

$$
\left.\left(\frac{d \lambda}{d \tau_{2}}\right)^{-1}=\frac{3 \lambda^{2}+2 \lambda\left(A_{1}+B_{2}\right)+B_{1}+E_{2}}{\lambda\left[H-B_{3}-\left\{\lambda^{3}+\lambda^{2}\left(A_{1}+B_{2}\right)+\lambda\left(B_{1}+E_{2}\right)\right\}\right.}\right]+\frac{2 \lambda G_{2}+E_{1}+G_{1}}{G_{2} \lambda^{3}+\lambda^{2}\left(E_{1}+G_{1}\right)}-\frac{\tau_{2}}{\lambda}
$$

by using

$$
e^{-\lambda \tau_{2}}=\frac{H-B_{3}-\left\{\lambda^{3}+\lambda^{2}\left(A_{1}+B_{2}\right)+\lambda\left(B_{1}+E_{2}\right)\right\}}{G_{2} \lambda^{2}+\lambda\left(E_{1}+G_{1}\right)} .
$$

We can obtain here,

$\left[\frac{d(\operatorname{Re} \lambda)}{d \tau_{2}}\right]_{\tau=\tau_{0}} \neq 0$.

We will verify the condition (3.18c) numerically. Shows that transversality condition holds and hence Hopf bifurcation occurs at $\tau_{2}=\tau_{20}$.

Case 2: $\tau_{1}=\tau_{2}=\tau>0$

Differentiating equation (3.11) with respect to $\tau$, we obtain

$$
\begin{gathered}
{\left[3 \lambda^{2}+2 \lambda A_{1}+E_{2}-2 \lambda E_{1} \tau e^{-2 \lambda \tau}+E_{1} e^{-2 \lambda \tau}-\tau e^{-\lambda \tau}\left\{\begin{array}{l}
\lambda^{2}\left(B_{2}+G_{2}\right)+ \\
\lambda\left(B_{1}+G_{1}\right)+B_{3}
\end{array}\right\}+e^{-\lambda \tau}\left\{\begin{array}{l}
2 \lambda\left(B_{2}+G_{2}\right)+ \\
B_{1}+G_{1}
\end{array}\right\}\right] \frac{d \lambda}{d \tau}} \\
=\lambda\left[e^{-\lambda \tau}\left\{\lambda^{2}\left(B_{2}+G_{2}\right)+\lambda\left(G_{1}+B_{1}\right)+B_{3}\right\}+2 E_{1} \lambda e^{-2 \lambda \tau}\right],
\end{gathered}
$$

therefore,

$$
\left(\frac{d \lambda}{d \tau}\right)^{-1}=\frac{3 \lambda^{2}+2 \lambda A_{1}+E_{2}+E_{1} e^{-2 \lambda \tau}+e^{-\lambda \tau}\left[2 \lambda\left(B_{2}+G_{2}\right)+B_{1}+G_{1}\right]}{\lambda\left[e^{-\lambda \tau}\left\{\left(B_{2}+G_{2}\right) \lambda^{2}+\left(B_{1}+G_{1}\right) \lambda+B_{3}\right\}+2 E_{1} \lambda e^{-2 \lambda \tau}\right]}-\frac{\tau}{\lambda} \text {. }
$$

We can obtain here,

$$
\left[\frac{d(\operatorname{Re} \lambda)}{d \tau}\right]_{\tau=\tau_{0}} \neq 0 \text {. }
$$

Verifying numerically it has been obtained that the transversality condition holds and hence Hopf bifurcation occurs at $\tau=\tau_{0}$. 


\section{Numerical simulation and discussion}

In this section, we present numerical simulation to illustrate results obtained in previous sections. The system (1) is integrated using fourth order Runge - Kutta Method with the help of MATLAB software package under the following set of parameters:

\begin{tabular}{|c|l|c|}
\hline Parameter & \multicolumn{1}{|c|}{ Meaning } & \multicolumn{1}{|c|}{ Value } \\
\hline$r_{1}$ & Intrinsic growth rate of the prey & 20 \\
\hline$r_{2}$ & Intrinsic growth rate of the predator & 15 \\
\hline$K_{1}$ & Carrying capacity of environment for prey & 1.8 \\
\hline$w$ & The attack rate of predator & 10 \\
\hline$D$ & The effect of the prey in evading a predator's attack & 10 \\
\hline$K_{2}$ & Carrying capacity of environment for predator & 0.4 \\
\hline$w_{1}$ & The maximal production efficiency of predator & 10 \\
\hline$D_{1}$ & The half-saturation constant & 0.2 \\
\hline$w_{2}$ & The saturation killing rate & 10 \\
\hline$D_{2}$ & The half-saturation constant & 0.13 \\
\hline$C$ & The rate of self-reproduction & 0.4 \\
\hline$w_{3}$ & The maximum predation rate & 2 \\
\hline$D_{3}$ & The residual reduction in the predator & \\
\hline
\end{tabular}

The interior equilibrium point of Eq. (1) with Table (a) is

Table (a) $E_{5}(14.8831,1.07692,1001.65)$.

Here, we are studying only for interior equilibrium point. By computation, we have $\delta_{0}=1.2451, \tau_{2_{0}}=0.49$. Here we show that the transversal condition (3.18c) is satisfied as:

$\left[\frac{d(\operatorname{Re} \lambda)}{d \tau_{2}}\right]_{\tau=\tau_{0}}=0.378658 \neq 0$.

Therefore, $\left(x^{*}, y^{*}, z^{*}\right)$ is asymptotically stable for $\tau_{2}<\tau_{2_{0}}=0.49$ and unstable for $\tau_{2}>\tau_{2_{0}}=0.49$. We can see this effect in Fig (1a) and Fig (1b). When $\tau_{2}=\tau_{2_{0}}$, Eq.(1) with data (a) undergoes a Hopf bifurcation at $\left(x^{*}, y^{*}, z^{*}\right)$, that is, there is an small amplitude periodic solution around $\left(x^{*}, y^{*}, z^{*}\right)$ when $\tau_{1}=0$ and $\tau_{2}$ is close to $\tau_{2_{0}}$, which is shown in Fig.(1a) and Fig.(1b). The transversal condition (3.19c) is also satisfied for Table (a) as $\left[\frac{d(\operatorname{Re} \lambda)}{d \tau}\right]_{\tau=\tau_{0}}=0.0048527 \neq 0$ for $\delta_{0}=1.98, \tau_{0}=0.75$. When $\tau_{1} \neq \tau_{2}, \tau_{1}>0, \tau_{2}>0$, we assume that $\tau_{1}=0.18$ and see the effect of $\tau_{2}$ on food chain model. This effect can be shown in Fig (2a) and Fig (2b). We also show here nature of system when there is no delay, see Fig. (3). When $\tau_{1}=\tau_{2}=\tau>0$, $\left(x^{*}, y^{*}, z^{*}\right)$ is asymptotically stable for $\tau<\tau_{0}=0.75$ and unstable for $\tau>\tau_{0}=0.75$. We can see this nature of the system in Fig. (4a) and Fig. (4b). 

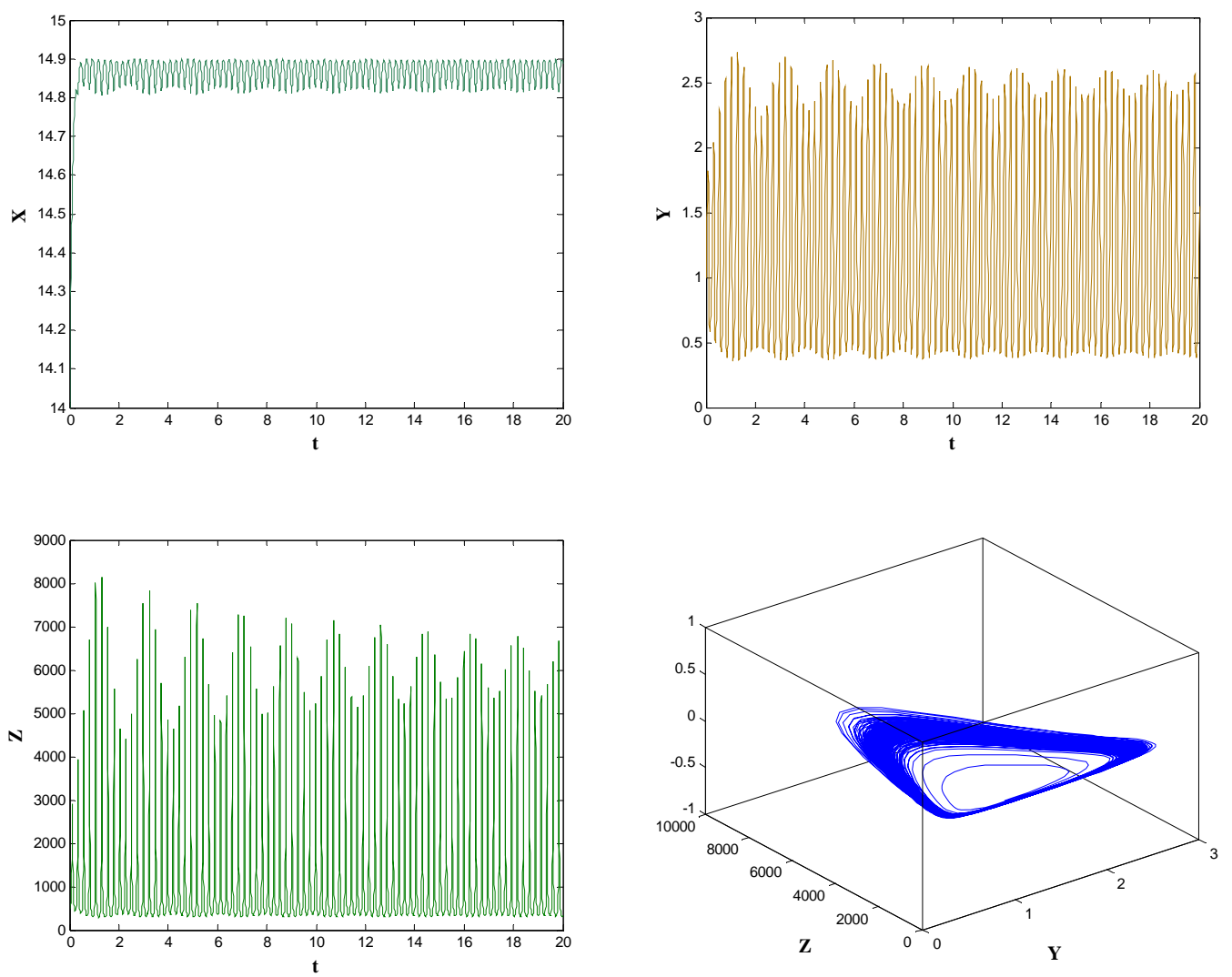

Fig(1a) when $\tau_{1}=0, \tau_{2}=0.50$
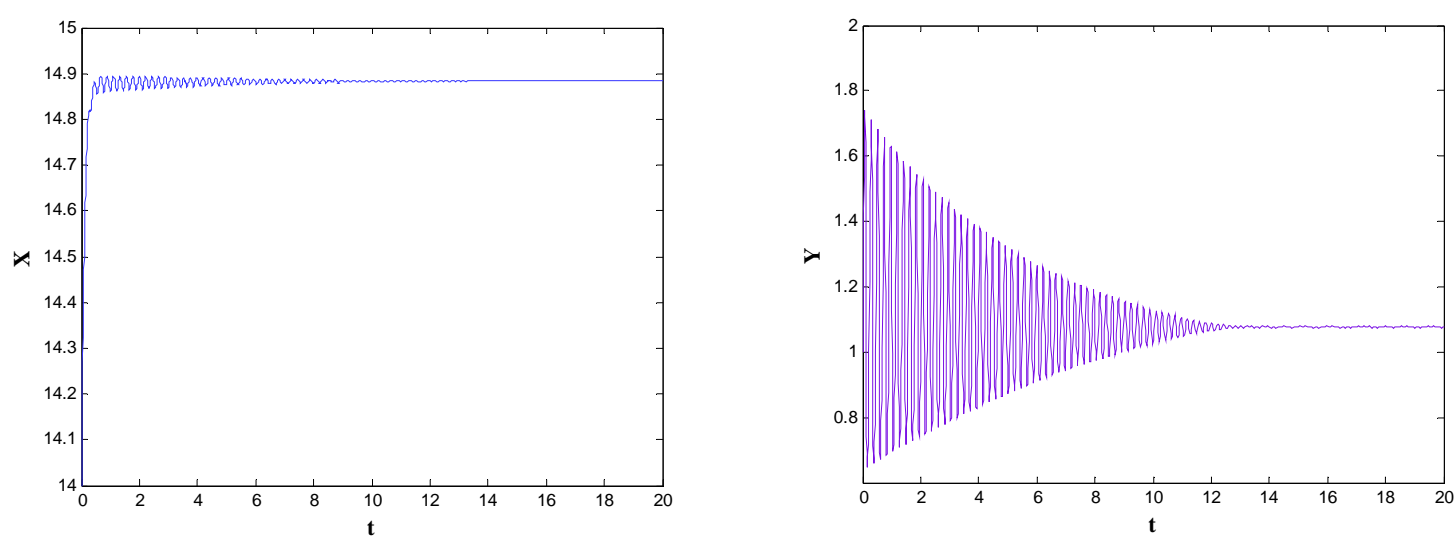

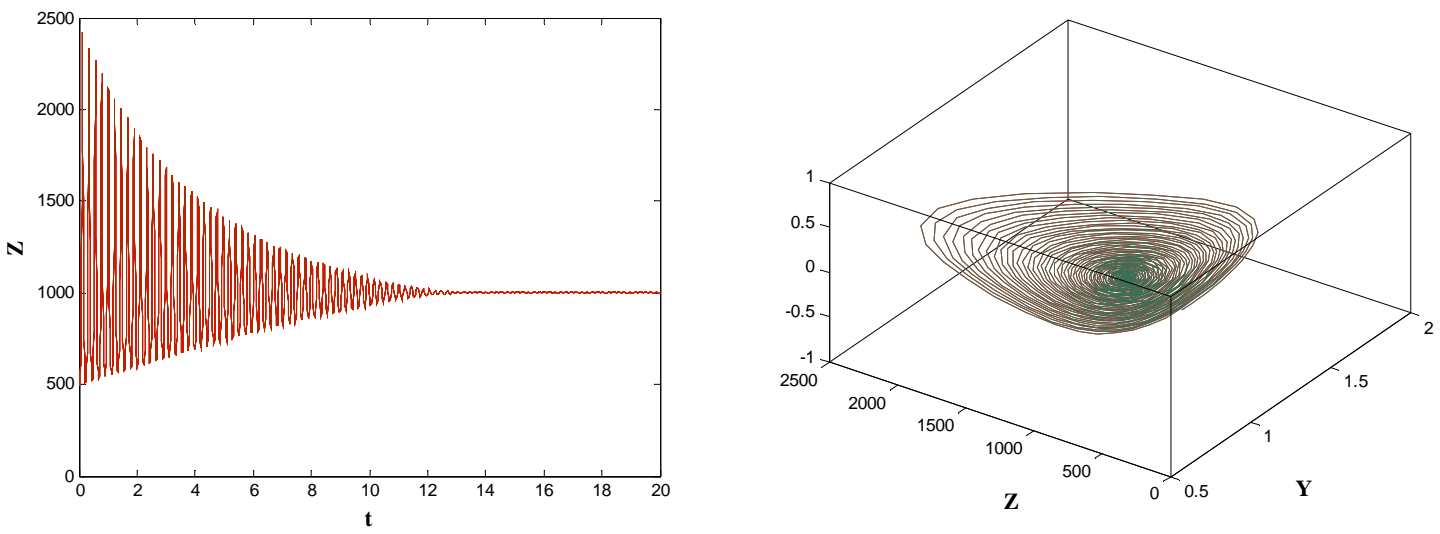

Fig. (1b) when $\tau_{1}=0, \tau_{2}=0.01$

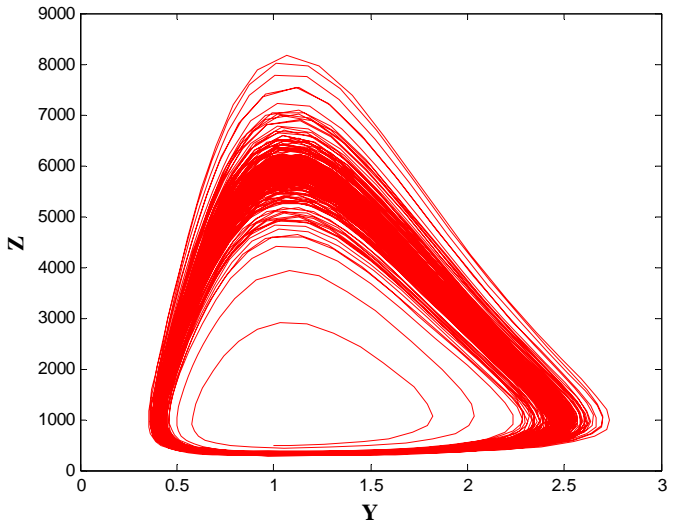

Fig. (2a) when $\tau_{1}=0.18, \tau_{2}=0.50$

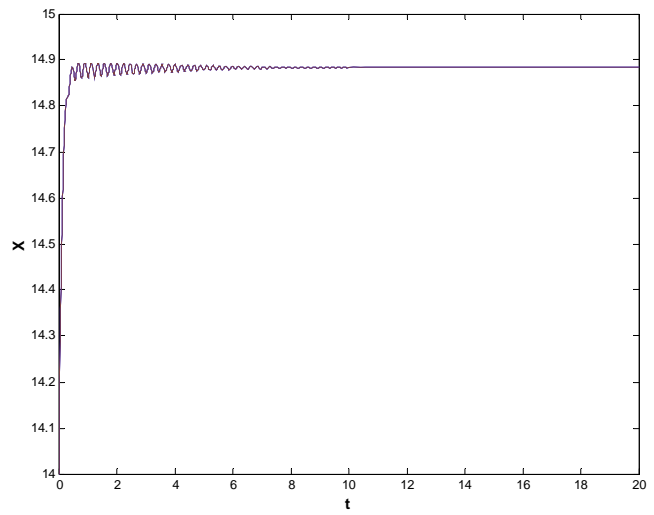

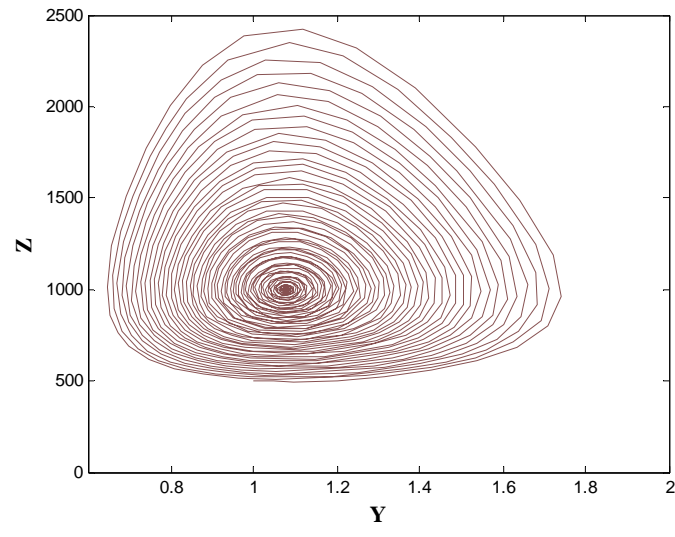

Fig. (2b) when $\tau_{1}=0.18, \tau_{2}=0.01$

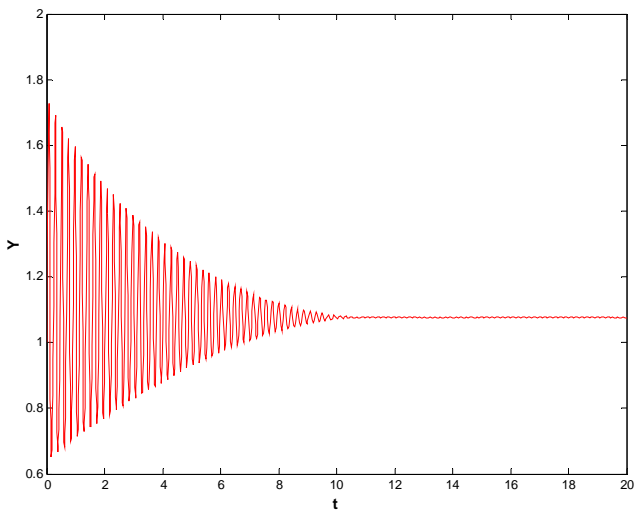



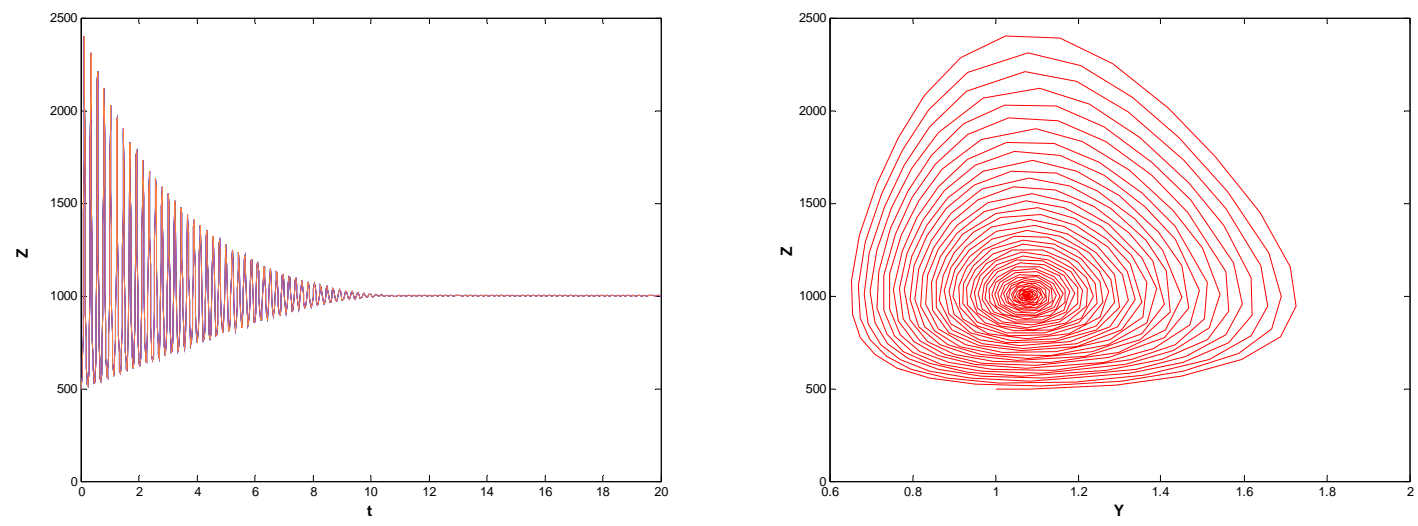

Fig.(3) when $\tau_{1}=0, \tau_{2}=0$

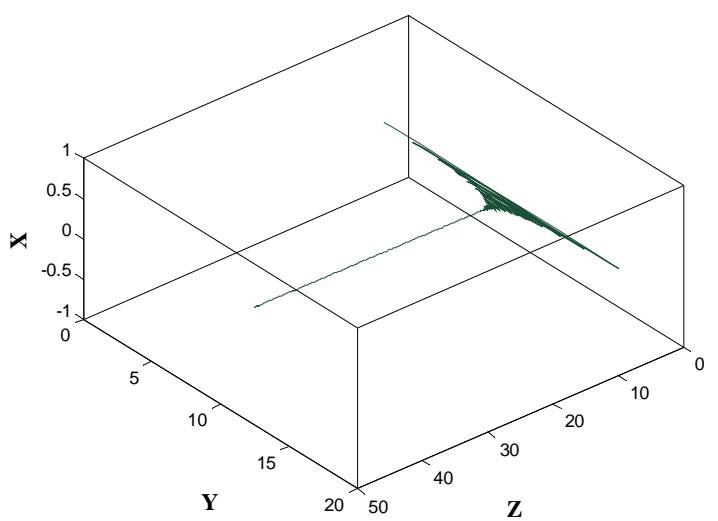

Fig.(4a) when $\tau_{1}=\tau_{2}=\tau=0.7$

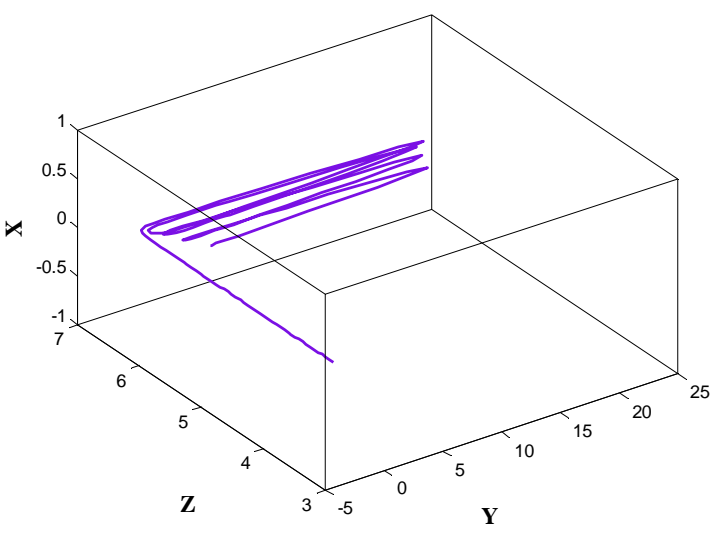

Fig.(4b) when $\tau_{1}=\tau_{2}=\tau=0.8$

\section{Conclusion}

Based on the food chain model proposed by Upadhyay and Rai (2001), we have proposed a food chain model with two delays, and investigated its dynamics. We have obtained the sufficient conditions for stability of interior equilibrium point. Moreover, conditions for the existence of Hopf bifurcation for the cases $\tau_{1}=0, \tau_{2}>0$ and $\tau_{1}=\tau_{2}=\tau>0$, respectively are determined. We have found critical value of delay and observed that under certain conditions, system bifurcates from the steady states of system (1) at some critical values of $\tau\left(\tau_{1}, \tau_{2}\right)$. Maturation delay always acts as a destabilizing factor. We have shown that maturation delay always satisfies the Hopf bifurcation condition. Hence, we conclude from our analysis that the stability properties of the system could switch with the time delay that is incorporated on different densities in the model.

\section{References}

Celik C.. 2008. The stability and Hopf bifurcation for a predator-prey system with time delay. Chaos, Solitons \& Fractals, Vol. 37, No. 1, pp. 87-99.

Celik C. and Duman O.. 2009. Allee effect in a discrete-time predator-prey system. Chaos, Solitons \& Fractals, Vol. 40, pp. 1956-62.

Chen X. 2007. Periodicity in a nonlinear discrete predator-prey system with state dependent delays. Nonlinear Anal RWA, Vol. 8, pp. 435-46.

Chiu C.H. and Hsu S.B.. 1998. Extinction of top predator in a three level food-chain model. J. Math. Biol., Vol. 37, pp. 372-380.

Cooke KL. and Grossman Z.. 1982. Discrete delay, distributed delay and stability switches. J Math Anal Appl., Vol. 86, No. 2, pp. 592-627. 
Freedman H.I. and Waltman P.. 1977. Mathematical analysis of some three-species food-chain models. Math. Biosci. , Vol. 33, pp. 257-276.

Freedman H.I.. 1980. Deterministic mathematical models in population ecology. New York, Marcel Dekker, 1980.

Freedman H.I. and So J.W.-H., 1985. Global stability and persistence of simple food chains, Math. Biosci. , Vol. 76, pp. 69-86.

Gopalsamy K. 1992. Stability and Oscillations in Delay Differential Equations of Population Dynamics. Kluwer Academics, Dordrecht.

Klebanoff and Hastings A.. 1993. Chaos in three species food chains. J. Math. Biol., Vol. 32, pp. 427-451.

Klebanoff and Hastings A.. 1994. Chaos in one-predator, two-prey models: general results from bifurcation theory. Math. Biosci. , Vol. 122, pp. 221-233.

Kuang Y.. 1993. Delay Differential Equations with Applications in Population Dynamics. Academic Press, New York.

Kuang Y. 1990. Global stability of Gause-type predator-prey systems. J. Math. Biol., Vol. 28, pp. 463 - 474.

Kuang Y.. 2001. Global stability and persistence in diffusive food chains. The ANZIAM J., Vol. 43, pp. 247-268.

Macdonald N. 1989. Biological Delay Systems: Linear Stability Theory. Cambridge Univ. Press, Cambridge.

McCann K. and Yodzis P.. 1995. Bifurcation structure of a three-species food chain model. Theor. Pop. Biol., Vol. 48, pp. 93-125.

May R.M. 1981. Theoretical Ecology. Principles and Applications, Second Ed., Blackwell, Oxford.

May. R.M. 2001. Stability and complexity in model ecosystems (Princeton Landmarks in Biology). Princeton, Princeton University Press.

Meng X.Y., Huo H. F. and Xiang H.. 2011. Hopf bifurcation in a three-species system with delays. J. Appl. Math. Comput., Vol. 35, pp. 635-661.

Muratori S. and Rinaldi S.. 1992. Low- and high-frequency oscillations in three-dimensional food chain systems. SIAM J. Appl. Math., Vol. 52, pp. 1688-1706.

Murray J.D.. 1989. Mathematical Biology. Springer-Verlag, New York.

Murray J.D.. 1993. Mathematical Biology. Second Corrected Ed., Springer, Heidelberg.

Takeuchi Y.. 1996. Global dynamical properties of Lotka - Volterra systems. World Scientific, Singapore.

Turchin. P. 2003. Complex population dynamics: A theoretical/empirical synthesis. Princeton University Press, Princeton, New Jersey.

Upadhyay R.K. and Rai. V. 2001. Crisis-limited chaotic dynamics in ecological systems. Chaos, Solitons and Fractals, Vol. 12, pp. 205-218.

Vance R.R. 1978. Predation and resource partitioning in one predation two prey communities. Am. Nat., Vol. 112, pp. 797-913.

Yu.A. Kuznetsov and Rinaldi S.. 1996. Remarks on food chain dynamics. Math. Biosci. , Vol. 134, pp. 1-33.

Yang Y.. 2009. Hopf bifurcation in a two-predator, one-prey system with time delay. Appl. Math. Comput., Vol. 214, pp. 228-235.

Yan X.P and Zhang C.H.. 2008. Hopf bifurcation in a delayed Lokta-Volterra predator-prey system. Nonlinear Anal., Real World Appl., Vol. 9, pp. 114-127.

\section{Biographical notes}

Manju Agarwal holds Ph.D. Degree in Applied Mathematics and currently she is working as a Professor and Head of Department of Mathematics and Astronomy, Lucknow University, Lucknow. Her areas of research interest are Mathematical Modeling, Environmental Pollution and Mathematical Ecology.

Rachana Pathak is doing Ph.D. under guidance of Prof. Manju Agarwal in Applied Mathematics. Her areas of research interest are Mathematical Modeling and Mathematical Ecology.

Received September 2011

Accepted December 2011

Final acceptance in revised form February 2012 